8. Lin GA, Lubinga S, Agboola F, Carlson J, et al. Prophylaxis for hereditary angioedema with lanadelumab and $\mathrm{Cl}$ inhibitors: effectiveness and value. Final evidence report. Institute for Clinical and Economic Review. November 15, 2018. Available at: https://icer-review.org/material/angioedema-final-report/. Accessed January 15, 2019.

9. Zuraw BL, Busse PJ, White M, et al. Nanofiltered $\mathrm{Cl}$ inhibitor concentrate for treatment of hereditary angioedema. N Engl J Med. 2010;363(6):513-22.

10. Aygören-Pürsün E, Soteres D, Moldovan D, et al. Preventing hereditary angioedema attacks in children using Cinryze: interim efficacy and safety phase 3 findings. Int Arch Allergy Immunol. 2017;173(2):114-19.

11. Zuraw BL, Kalfus I. Safety and efficacy of prophylactic nanofiltered Cl-inhibitor in hereditary angioedema. Am J Med. 2012;125(9):938.el-7.

12. Longhurst H, Cicardi M, Craig T, et al. Prevention of hereditary angioedema attacks with a subcutaneous $\mathrm{Cl}$ inhibitor. $\mathrm{N}$ Engl J Med. 2017;376(12):1131-40
13. Lumry WR, Miller DP, Newcomer S, Fitts D, Dayno J. Quality of life in patients with hereditary angioedema receiving therapy for routine prevention of attacks. Allergy Asthma Proc. 2014;35(5):371-76.

14. Banerji A, Riedl M, Bernstein J, et al. Lanadelumab for prevention of attacks in hereditary angioedema: results from the phase 3 help study. Ann Allergy Asthma Immunol. 2017;119(5):S5.

15. Pearson SD. The ICER value framework: integrating cost effectiveness and affordability in the assessment of health care value. Value Health. 2018;21(3):258-65.

16. Institute for Clinical and Economic Review. ICER value assessment framework. Available at: https://icer-review.org/methodology/icers-methods/ icer-value-assessment-framework/. Accessed January 15, 2019.

\title{
Cost-Effectiveness of Prophylactic Medications for the Treatment of Hereditary Angioedema Due to C1 Inhibitor Deficiency: A Real-World U.S. Perspective \\ Bruce L. Zuraw, MD
}

\section{COMMENTARY}

due to $\mathrm{Cl}$ inhibitor deficiency (HAE-ClINH) is a rare but serious disease that may be associated with substantial morbidity or even mortality. ${ }^{1}$ The unraveling of the basic pathophysiology over the past 4 decades has resulted in the development and licensing of 7 safe and effective medications for the treatment of HAE-CIINH. These treatments can be broadly divided into 2 categories: those used

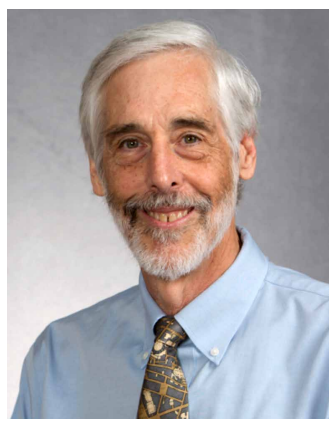

Management of HAE-ClINH patients has become increasingly complex as the therapeutic options have expanded. Furthermore, third-party payers are struggling to develop appropriate criteria for evaluating which medications will cover which patients. Given the burgeoning treatment options available, there is clearly a need to better define the cost-effectiveness of these medications. The final report on HAE medications from the Institute for Clinical and Economic Review (ICER) is an on-demand to treat angioedema attacks once they have begun, and those used prophylactically to prevent angioedema attacks from occurring.

All patients with HAE-CIINH need to have effective ondemand medications available in case of an attack. Some patients can be managed with on-demand medications alone, while other patients do better on long-term prophylactic treatment. There are no firm criteria for choosing which patients should be treated with a prophylactic medication. Most guideline and consensus papers recommend that treatment be individualized based on a given patient's specific history and needs. ${ }^{2-4}$ ambitious effort to provide such a definition, using a rigorous pharmacoeconomic approach to calculate the cost-effectiveness of the current modern prophylactic medications Cinryze and Haegarda (ClINHs) and lanadelumab (Takhzyro).

Starting with a literature review, the ICER authors estimated HAE-CIINH patient clinical characteristics and costs and then used a Markov model calculated on a monthly cycle to estimate lifetime costs of treatment with on-demand medications alone versus treatment with a prophylactic plus on-demand medications. Estimated costs of treatment were then analyzed in relationship to estimated changes in quality-adjusted lifeyears (QALYs) in an attempt to derive the cost-effectiveness of the treatments. The authors also performed a sensitivity end, they concluded that the currently approved prophylactic analysis that evaluated multiple clinical scenarios. In the 


\section{Cost-Effectiveness of Prophylactic Medications for the Treatment of Hereditary Angioedema Due to C1 Inhibitor Deficiency: A Real-World U.S. Perspective}

medications were not cost-effective for the majority of HAE$\mathrm{ClINH}$ patients. Haegarda was considered to give high longterm value for money versus on-demand therapy by only 1 of 15 voting members, while lanadelumab and Cinryze received no votes for giving high value.

While this effort to develop a pharmacoeconomic model for the treatment of HAE-ClINH is laudable, the validity of the underlying assumptions and the real-world clinical significance of the results need to be addressed. This commentary offers a critique of the medical relevance of the ICER findings, as well as how the ICER recommendations fit with our current understanding of optimal HAE-ClINH management. The goal is to provide readers with the appropriate context in which to interpret the ICER results. This critique focuses on 3 questions:

- Are the underlying clinical assumptions about HAE-ClINH used in this analysis valid?

- Do the QALY estimates accurately capture the actual benefit from prophylactic treatments?

- Is this pharmacoeconomic analysis consonant with the ethical principles of medical decision making?

By addressing these questions, I hope to illuminate how physicians and third-party payers should interpret the recommendations of the ICER analysis for the treatment of patients with HAE-CIINH.

\section{Underlying Assumptions}

The dearth of high-quality unequivocal data to use in a pharmacoeconomic model for HAE-ClINH is not unexpected for a rare disease. Nevertheless, there is reason to question some of the key clinical assumptions made by the ICER team. While many additional clinical assumptions could be challenged, comments are restricted to those assumptions that have a major impact on the cost-effectiveness model and that represent an error of interpretation rather than a disagreement about the magnitude of a clinical parameter.

First, the ICER study assumed that all moderate and severe attacks will be treated with on-demand medications. This assumption ignores 2 key recommendations contained in multiple consensus and guideline papers that reflect the widely accepted clinical goal to minimize patient morbidity and mortality. These recommendations are as follows: all attacks should be eligible for treatment, and attacks should be treated as early as possible. Patients are therefore instructed to treat their attacks before it becomes clear whether the attack will end up being mild, moderate, or severe because once the attack becomes moderate or severe, the patient will by definition suffer morbidity. These 2 recommendations have resulted in a situation in the United States where many or most mild attacks are treated with on-demand medications. Based on this, the ICER study likely significantly underestimates the percentage of attacks that will be treated with costly on-demand medications.
Second, the retreatment rate among patients using ondemand medications must be questioned. For example, the ICER model used a retreatment rate of $12.7 \%$ for icatibant based on experience in Italy. ${ }^{5}$ Interestingly, a European report on the effectiveness of icatibant found that the retreatment rate was highly variable among different countries, ranging from $21.6 \%$ in France to $2.4 \%$ in Germany-Austria. ${ }^{6}$ In a recent unpublished survey involving HAE-ClINH patients from the United States, a retreatment rate of $29 \%$ was found. These results suggest that the costs of on-demand treatment with icatibant (and likely other on-demand medications) will likely be far higher in the United States than estimated in the ICER model.

Third, the assumption that there would be no difference in survival among the strategies is deeply flawed, since HAE-ClINH patients continue to die even with modern on-demand medications available. While the percentage of attacks that result in death is now, thankfully, low, it is not zero. The risk of death is a direct function of the total number of attacks, which is markedly greater in patients receiving only on-demand medications than in patients receiving prophylactic treatment. The excess societal and personal costs from premature deaths of HAE-ClINH patients (including children and young adults) receiving only on-demand medications are not included in the ICER analysis.

Fourth, the ICER authors correctly identify the effect of prophylactic treatment on attack rate reduction in the pivotal phase 3 clinical trials. Nevertheless, using these figures leads to serious errors in the overall analysis because these rates reflect the particular constraints of phase 3 pivotal trials rather than real-world effectiveness. For example, Cinryze resulted in a $51 \%$ reduction of attacks in the phase 3 clinical trial, ${ }^{7}$ but a $90 \%$ reduction in attacks was found in the open-label extension study. ${ }^{8}$ Similarly, $40 \%$ of patients receiving $60 \mathrm{IU} / \mathrm{kg}$ of Haegarda were attack-free in the 14 weeks of the clinical study, ${ }^{9}$ but $83 \%$ were attack-free during the subsequent 24 weeks after 18 months of use in the open-label extension (Craig et al., unpublished data, September 7, 2018). A lanadelumab study reported that $44 \%$ of patients receiving $300 \mathrm{mg}$ every 2 weeks were attack-free during the clinical study, but a post hoc subanalysis during the time when the drug was at steady-state revealed that $77 \%$ of patients were attack-free. ${ }^{10}$

In clinical practice, we routinely see that almost all of our patients taking either Haegarda or lanadelumab are attack-free. However, the ICER analysis used attack rates of approximately 1.68 attacks per month, 0.54 attacks per month, and 0.44 attacks per month for patients receiving Cinryze, Haegarda, or lanadelumab, respectively. The reality of $0-1$ attacks per year in almost all HAE-ClINH patients taking these medications would have a profound effect on the cost-effectiveness analysis.

\section{Quality-Adjusted Life-Years}

All costs of the various treatment strategies are ultimately evaluated against the benefit that those strategies bring. In the 
ICER analysis, the authors used QALY to determine cost-effectiveness by dividing the total costs by the number of QALYs. Therefore, the measurement of QALY used will have an enormous effect on the cost-effectiveness conclusions.

The measure chosen in the ICER analysis is based on a small study (101 subjects) from Sweden, using the EuroQoL 5D (EQ-5D) to model the benefit of treatment on quality of life. ${ }^{11}$ The EQ-5D is a validated instrument for measuring quality of life, consisting of 5 questions regarding mobility, self-care, usual activities, pain/discomfort, and anxiety/depression. Unfortunately, the EQ-5D does not address the unique and specific factors that strongly influence quality of life for HAE patients. A comparison between the EQ-5D and an angioedema-specific quality of life instrument revealed multiple differences that could have a significant effect on the analysis. ${ }^{12}$ Angioedema-specific, as well as HAE-specific, quality of life instruments have been developed and validated but were not used for this analysis. ${ }^{13,14}$

A greater concern is the relatively modest improvement in QALY assigned to patients treated with prophylactic medications in this model. The number of QALYs rose from 17.47 in the no prophylaxis group to between 18.21 and 18.66 in the groups receiving prophylaxis. For many reasons, including those already mentioned, the clinical effectiveness of long-term prophylaxis has been vastly and systematically underestimated in the ICER analysis, resulting in only small improvements in QALYs. As an example, the lanadelumab phase 3 trial showed a profound improvement in angioedema-specific quality of life in lanadelumab-treated subjects compared with subjects treated on-demand only over the course of a relatively short (26-week) study. ${ }^{10}$

The ICER analysis is also incompatible with consistent comments on effective prophylactic medications from HAE-ClINH patients who are seen at our clinic. These patients report that their lives have dramatically changed for the better and that they feel normal for the first time. The ICER model for estimating quality of life assessed the effect of patient age, as well as the predicted number and severity of attacks, but failed to account for the sense of well-being that patients experience from effective prophylactic treatment when they feel normal and no longer fear unpredictable attacks. Since cost-effectiveness is calculated by the cost per QALY gained, accuracy in estimating the expected improvement in quality of life is of utmost importance.

\section{Ethical Issues}

The third area that will be briefly touched upon is the effect of the ICER recommendations on the ethical issues that physicians face when recommending a treatment plan. The validity and importance of using cost-effectiveness as a tool in medical decision making is not in question. Medical decision making, however, is deeply based on the ethical principles of beneficence and justice, and the physician is obligated to consider the harm that could result from a treatment decision.

When considering the relative weights of these potentially competing imperatives, the strength of the underlying evidence must be considered. As already noted, there are serious concerns about the validity of the ICER pharmacoeconomic analysis. In contrast, HAE-ClINH is clearly recognized as a severe and unpredictable disease that can result in death, even among children and adolescents. Failure to use a treatment that can absolutely reduce and potentially eliminate the risk of this ultimate harm may therefore be viewed as an abrogation of the physician's responsibility to treat the patient in a manner that protects the patient from serious harm.

\section{ICER Recommendations in the Real World}

The management of patients with HAE-ClINH has become very complex and costly. Because it is a rare and highly variable disease, there are no head-to-head comparisons between the available medications used to treat HAE-CIINH. It is becoming increasingly clear that HAE-ClINH patients need to have expert physicians involved in their care, who fully understand the disease and its treatment. Even with the involvement of expert physicians, additional data about cost-effectiveness of the different treatment options are needed.

This report from ICER raises awareness of the importance of developing a clearer understanding of the costs and benefits of the many options now available to U.S. physicians who treat these patients. While such pharmacoeconomic data is clearly needed, the ICER analysis has serious flaws in the underlying assumptions and the assessment of benefit. There were also substantial errors in estimating the costs of HAE-ClINH care in the United States beyond direct drug costs. These underestimates involve direct costs (particularly emergency room visits, hospitalizations, and intubations or cricothyrotomies); indirect costs of attacks; and productivity costs. For all of these reasons, the ICER analysis does not offer clinically useful costeffectiveness information that can be relied on to inform either physician or payer decision maker.

Many of the problems identified in this critique can and should be corrected in subsequent studies. Future pharmacoeconomic analyses of prophylactic treatments would benefit by incorporating 2 key HAE-ClINH realities. First, the overwhelming medical, personal, societal, and ethical costs of HAE attacks need to be fully recognized. Second, the efficacy of prophylactic treatment has reached the point where HAEClINH patients can expect to have few or no attacks. The need to individualize treatment for each patient will remain, and physicians will need to determine which prophylactic medication achieves the desired end result for a given patient. These realities portend a paradigm shift that has profound implications for the treatment of HAE-ClINH, patient quality of life, and cost-effectiveness of long-term prophylaxis. 


\section{Cost-Effectiveness of Prophylactic Medications for the Treatment of Hereditary Angioedema Due to C1 Inhibitor Deficiency: A Real-World U.S. Perspective}

\section{Conclusions}

We now have 3 modern prophylactic medications approved for HAE-CIINH, and a number of new drugs are in various stages of development. There is every reason to believe that the benefits of prophylactic treatment of HAE-CIINH will continue to grow as more patients gain access to these medications, in part based on recognition that they are cost-effective. Given the right approach to treatment, we should soon end up in a virtuous cycle where patients with HAE-ClINH can expect to live entirely normal lives using medications that are truly cost-effective.

\section{Authors}

BRUCE L. ZURAW, MD, Division of Rheumatology, Allergy and Immunology, Department of Medicine, University of California San Diego School of Medicine and Medicine Service, San Diego VA Healthcare.

AUTHOR CORRESPONDENCE: Bruce L. Zuraw, MD, 9500 Gilman Dr., Mailcode \#0732, La Jolla, CA 92093. Tel.: 858.822.6597; E-mail: bzuraw@ucsd.edu.

\section{DISCLOSURES}

No funding supported the writing of this commentary. The author reports personal fees from BioCryst, CSL Behring, Shire, and Pharming and grants from Ionis. He is chair of the US HAEA Medical Advisory Board and scientific advisor for HAE International.

\section{REFERENCES}

1. Zuraw BL. Clinical practice. Hereditary angioedema. N Engl J Med. 2008:359(10):1027-36
2. Zuraw BL, Banerji A, Bernstein JA, et al. US Hereditary Angioedema Association Medical Advisory Board 2013 recommendations for the management of hereditary angioedema due to $\mathrm{Cl}$ inhibitor deficiency. J Allergy Clin Immunol Pract. 2013;1(5):458-67.

3. Maurer M, Magerl M, Ansotegui I, et al. The international WAO/EAACI guideline for the management of hereditary angioedema-the 2017 revision and update. Allergy. 2018;73(8):1575-96.

4. Betschel S, Badiou J, Binkley K, et al. Canadian hereditary angioedema guideline. Allergy Asthma Clin Immunol. 2014;10(1):50.

5. Zanichelli A, Mansi M, Azin GM, et al. Efficacy of on-demand treatment in reducing morbidity in patients with hereditary angioedema due to $\mathrm{Cl}$ inhibitor deficiency. Allergy. 2015;70(12):1553-58.

6. Caballero T, Aberer W, Longhurst HJ, et al. The Icatibant Outcome Survey: experience of hereditary angioedema management from six European countries. J Eur Acad Dermatol Venereol. 2017;31(7):1214-22.

7. Zuraw BL, Busse PJ, White M, et al. Nanofiltered Cl inhibitor concentrate for treatment of hereditary angioedema. N Engl J Med. 2010;363(6):513-22.

8. Zuraw BL, Kalfus I. Safety and efficacy of prophylactic nanofiltered Cl-inhibitor in hereditary angioedema. Am J Med. 2012;125(9):938.el-7.

9. Longhurst H, Cicardi M, Craig T, et al. Prevention of hereditary angioedema attacks with a subcutaneous $\mathrm{Cl}$ inhibitor. N Engl J Med. 2017;376(12):1131-40.

10. Banerji A, Riedl MA, Bernstein JA, et al. Effect of lanadelumab compared with placebo on prevention of hereditary angioedema attacks: a randomized clinical trial. JAMA. 2018;320(20):2108-21.

11. Nordenfelt P, Dawson S, Wahlgren CF, Lindfors A, Mallbris L, Bjorkander J. Quantifying the burden of disease and perceived health state in patients with hereditary angioedema in Sweden. Allergy Asthma Proc. 2014:35(2):185-90.

12. Nordenfelt P. Hereditary angioedema in Sweden: a national project. Department of Clinical and Experimental Medicine, Faculty of Medicine and Health Sciences, Linköping University; 2017:117.

13. Weller K, Groffik A, Magerl M, et al. Development and construct validation of the angioedema quality of life questionnaire. Allergy. 2012;67(10):1289-98

14. Prior N, Remor E, Gomez-Traseira C, et al. Development of a diseasespecific quality of life questionnaire for adult patients with hereditary angioedema due to $\mathrm{Cl}$ inhibitor deficiency (HAE-QOL): Spanish multi-centre research project. Health Qual Life Outcomes. 2012;10:82. 